\title{
Discretization Errors Associated with Reproducing Kernel Methods: One-Dimensional Domains *
}

\author{
Thomas E. Voth ${ }^{\dagger}$. \\ Thermal/Fluids Engineering Department \\ and \\ Mark A. Christon \\ Computational Physics R\&D Department
}

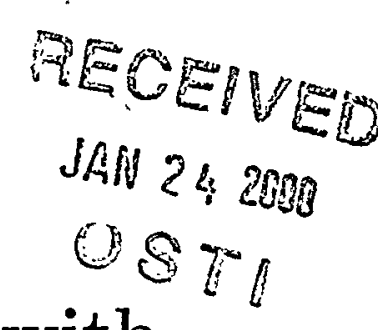

Computational Physics R\&D Department

Sandia National Laboratories

M/S 0836, P.O. Box 5800

Albuquerque, New Mexico 87185-0835

PH.: (505) 844-6004

FAX: (505) 844-8251

E-mail: tevoth@sandia.gov

submitted to:

Computer Methods in Applied Mechanics and Engineering

December 3, 1999

*Sandia is a multiprogram laboratory operated by Sandia Corporation, a Lockheed Martin Company, for the United States Department of Energy under Contract DE-AC04-94ALS5000.

†Corresponding Author 


\section{DISCLAIMER}

This report was prepared as an account of work sponsored by an agency of the United States Government. Neither the United States Government nor any agency thereof, nor any of their employees, make any warranty, express or implied, or assumes any legal liability or responsibility for the accuracy, completeness, or usefuiness of any information, apparatus, product, or process disclosed, or represents that its use would not infringe privately owned rights. Reference herein to any specific commercial product, process, or service by trade name, trademark, manufacturer, or otherwise does not necessarily constitute or imply its endorsement, recommendation, or favoring by the United States Government or any agency thereof. The views and opinions of authors expressed herein do not necessarily state or reflect those of the United States Government or any agency thereof. 


\section{DISCLAIMER}

Portions of this document may be illegible in electronic image products. Images are produced from the best available original document. 


\begin{abstract}
The Reproducing Kernel Particle Method (RKPM) is a discretization technique for partial differential equations that uses the method of weighted residuals, classical reproducing kernel theory and modified kernels to produce either "mesh-free" or "mesh-full" methods. Although RKPM has many appealing attributes, the method is new, and its numerical performance is just beginning to be quantified. In order to address the numerical performance of RKPM, von Neumann analysis is performed for semi-discretizations of three model one-dimensional PDEs. The von Neumann analyses results are used to examine the global and asymptotic behavior of the semi-discretizations.

The model PDEs considered for this analysis include the parabolic and hyperbolic (first and second-order wave) equations. Numerical diffusivity for the former and phase speed for the later are presented over the range of discrete wavenumbers and in an asymptotic sense as the particle spacing tends to zero. Group speed is also presented for the hyperbolic problems. Excellent diffusive and dispersive characteristics are observed when a consistent mass matrix formulation is used with the proper choice of refinement parameter. In contrast, the row-sum lumped mass matrix formulation severely degraded perfomance. The asymptotic analysis indicates that very good rates of convergence are possible when the consistent mass matrix formulation is used with an appropriate choice of refinement parameter.
\end{abstract}

Key Words: reproducing kernel particle methods, meshless, discretization errors

\title{
1 Introduction
}

The accurate simulation of diffusion, wave propagation or advection processes using discrete numerical schemes hinges upon having a clear understanding of the constraining numerical errors, the grid resolution to minimize these errors, and sufficient computational resources to effect solutions with the requisite grid scale. Examples of this may be seen when attempting to simulate solid-liquid phase change or ablation, model wave propagation in an acoustic medium, or compute turbulent flow fields via direct numerical simulation (DNS) or large eddy simulation (LES). In general, the computation of diffusion and wave propagation problems is limited by the wavelength that the grid can accurately represent. For example, controlling the dispersive errors to within $5 \%$ for finite difference or lumped-mass finite element semi-discretizations of the first-order wave equation requires approximately $11-12$ grid points per wavelength (see Table 2.6.2 in [7]).

An alternative to traditional grid-based approaches is the class of methods based on moving least-squares, reproducing kernels, and partitions of unity. An overview of the development of this class of numerical methods is presented by Belytschko, et al. [1]. The methods based upon reproducing kernels are of interest here because they promise to deliver enhanced numerical performance on a broad range of physical problems and provide a framework for multi-scale analyses. 
Liu and his co-workers have been developing reproducing kernel particle methods (RKPM) for a number of years and have demonstrated applications ranging from structural acoustics to large deformation mechanics problems $[14,15,16,19]$. In addition, Liu, et al. $[13,21]$ have combined reproducing kernel ideas with multi-resolution analysis using pre-wavelets, permitting the decomposition of discrete solutions into multiple scales. The application of RKPM to structural dynamics has been demonstrated by Liu, et al. [18] in addition to showing that the reproducing kernel interpolation functions satisfy consistency conditions. Uras, et al. [23] have applied RKPM to acoustics problems demonstrating that the dilation parameter in the window function may be used to perform the RKPM analog of $h-p$ adaptivity.

In a series of papers by Liu and $\mathrm{Li}[10,12,20]$ moving least-squares reproducing kernel methods are developed beginning with the basic formulation and continuing through a Fourier analysis and the incorporation of wavelet packets. The possibility for RKPM to deliver synchronized rates of convergence for the discrete functions and their derivatives has also been explored by Li and Liu [11]. The application of RKPM for nearly incompressible, hyper-elastic solids was considered by Chen, et al. [3], while the treatment of large deformation problems has been explored by Liu and his colleagues $[9,17]$. The enrichment of finite element computations with RKPMI has also been addressed, permitting local regions of the computational domain to be treated with RKPMI while the global problem is treated with a standard finite element formulation $[4,22]$.

In general, the application of discrete methods to parabolic and hyperbolic differential equations results in errors that distort the discrete solution relative to the true physics. For example, the discretization of a parabolic PDE results in an effective diffusivity that is a function of wavelength where the physical diffusivity is wavelength independent. In the context of heat conduction, the diffusivity is a measure of the ability of a medium to conduct relative to its ability to store thermal energy. An initial temperature distribution imposed in a diffusive medium will tend to diffuse at a rate set by its wavelength independent diffusivity. The effect of discretization is to make the diffusivity a function of the wavelength, modifying the diffusion rates of the individual Fourier components relative to the their physical rates, resulting in an error in the predicted time-dependent thermal solution.

For hyperbolic problems, the application of discrete methods results in solutions that are dispersive even though the physical phenomena is non-dispersive. Dispersion errors are typically characterized by the differences between the apparent numerical phase and group speed of waves and their true, or exact, counterparts. For linear acoustics, phase speed is the speed at which individual waves propagate while group speed is the speed of the energy associated with a wave packet $[7,24,25]$.

The study of diffusive and dispersive errors associated with discrete solutions is not new and has been used by numerous researchers to characterize the performance of numerical methods in the past. A brief review of the errors associated with hyperbolic problems is presented by Voth and Christon [5]. Studies of diffusive errors associated with the semi-discretizations of parabolic problems have been performed by Gresho et 
al. $[6,7]$, and Vichnevetsky et al. $[24,25,26]$.

Although the application of RKPM has been demonstrated on a broad class of problems, its numerical performance has not been fully investigated. In the discussion that follows, diffusion, dispersion and rates of convergence for several RKPM formulations of the parabolic and hyperbolic model PDEs are investigated. The discussion begins with an overview of the von Neumann and asymptotic error analysis and a summary of the formulae for computing the effective diffusivities, phase and group speed and rate of convergence for RKPM semi-discretizations of the model equations. In $\S 3$, the results of the von Neumann analyses are presented. Here, effective diffusivity and phase and group speed for consistent, lumped, and higher-order mass matrices are presented. Also investigated are the effect of integration rule and kernel function width. The discussion of the von Neumann results is followed by a presentation of the accuracy-in-the-small. Finally, the results are summarized and conclusions drawn.

\section{Formulation}

This section begins with a brief overview of the RKPM method. A detailed presentation of RKPM is beyond the scope of this paper, and the reader is directed to the literature for details concerning the method $[10,11,15,16]$. The formulae required for computing the normalized effective (numerical) diffusivity, phase speed and group speed follow the overview.

\subsection{Reproducing Kernel Particle Formulation}

For the sake of clarity, the following orerview is limited to one spatial dimension with uniform particle spacing although the formulation and analysis may be extended to higher dimensions with irregular particle distributions $[3,15,16]$. The RKPM[ formulation begins with the notion of a continuous kernel approximation of a function, $U$ on a domain $\Omega$,

$$
U^{R, a}(x)=\int_{\Omega} U(\xi) \varphi^{a}(x-\xi) d \xi,
$$

where $\varphi^{a}$ is the kernel function, $a$ is the dilation parameter that defines the width of the function and $U^{R, a}$ is a continuous approximation to $U$ at some scale defined by $a[15,19]$. In order to address discrete problems, numerical quadrature (i.e., trapezoidal or particle integration) is used to evaluate Eq. (1) as

$$
U^{a}(x)=\sum_{i=1}^{n} d_{i} \varphi^{a}\left(x-x_{i}\right) \Delta x_{i},
$$

where $n$ is the total number of particles in $\ddot{\Omega}, U^{a}$ is the discrete analog of $U^{R, a}, \Delta x_{i}$ is the variational volume and $d_{i}$ the coefficient associated with particle $i$ [15]. In general, $d_{i}$ is different from the value of the function at particle $i$ because the RKPM basis is non-nodal (i.e., it does not posses the Kronecker-delta property). 
One of the most commonly used kernel functions is the cubic spline. In one-dimension, the cubic spline kernel function is

$$
\varphi^{a}(z)= \begin{cases}\frac{2}{3 r \Delta x}\left[1-\frac{3}{2} z^{2}+\frac{3}{4} z^{3}\right] & z<1 \\ \frac{1}{6 r \Delta x}[2-z]^{3} & 1 \leq z<2 \\ 0 & z \geq 2\end{cases}
$$

where $z=\left|x-x_{i}\right| /(r \Delta x), x_{i}$ is the position of particle $i$ and $r$ is the refinement parameter $[1,15,16]$. On a grid of uniformly spaced particles, $r$ is related to the dilation parameter, $a$, as $r=a / \Delta x$. Hence, as with the dilation parameter, the refinement parameter permits dilation of the kernel function, and subsequently, its domain of influence. In the remaining discussion, the superscript $a$ is omitted for conciseness.

In general, Eq. (2) will not exactly reproduce an arbitrary polynomial. The accurate reproduction of polynomials to degree $p$ is guaranteed by introducing a modified kernel function,

$$
\bar{\varphi}\left(x-x_{i}\right)=\sum_{k=0}^{p} \beta_{k}(x) \varphi\left(x-x_{i}\right)\left(x-x_{i}\right)^{k},
$$

where $\beta_{k}(x)$ is a set of correction functions that vary within the domain $\Omega[15,16]$. The modified kernel function replaces $\varphi$ in Eq. (2) yielding

$$
U(x)=\sum_{i=1}^{n} d_{i} \bar{\varphi}\left(x-x_{i}\right) \Delta x_{i} .
$$

The correction functions are determined by substituting Eq. (4) into Eq. (5) and requiring that the resulting kernel approximation reproduce polynomials to the desired degree. For linear consistency, the following constraints are required.

$$
\begin{gathered}
\sum_{i=1}^{n}\left[\beta_{0}(x)+\beta_{1}(x)\left(x-x_{i}\right)\right] \varphi\left(x-x_{i}\right) \Delta x_{i}=1 \quad \text { and }, \\
\sum_{i=1}^{n}\left[\beta_{0}(x)+\beta_{1}(x)\left(x-x_{i}\right)\right] \varphi\left(x-x_{i}\right) x_{i} \Delta x_{i}=x .
\end{gathered}
$$

From these equations, $\beta_{0}(x)$ and $\beta_{1}(x)$ may be calculated in a point-wise fashion in the domain.

In the reproducing kernel particle method, shape functions, $N_{i}(x)$, arise by considering Eq. (5) to be an expansion in terms of $N_{i}(x)$ where

$$
N_{i}(x)=\bar{\varphi}\left(x-x_{i}\right) \Delta x_{i} .
$$

These "shape functions" may be used directly in a Bubnov-Galerkin procedure to obtain the weak form of the model PDEs yielding mass, stiffness and advection matrices.

In the formulation of the semi-discrete equations, the spatial derivatives of $N_{i}(x)$ are required. Calculation of these derivatives requires the computation of the derivatives of $\beta_{k}(x)$ as well. Although the calculation of these derivatives is rather straight forward, the algebra required is significant. For this reason, the details are omitted here, and the reader may wish to consult the work of Liu, et al. $[15,16]$ 


\section{2 von Neumann Analysis}

With the RKPM formulation outlined, the weak forms of three model partial differential equations (two hyperbolic and one parabolic) are presented along with a description of the Fourier analysis. For generality, the numerical dispersion and diffusion relations are generated for the the two-dimensional model equations from which the one-dimensional counterparts are obtained.

To begin, the two-dimensional first-order wave, second-order wave and parabolic partial differential equations are, in Cartesian coordinates,

$$
\begin{gathered}
\frac{\partial U}{\partial t}+c_{x} \frac{\partial U}{\partial x}+c_{y} \frac{\partial U}{\partial y}=0 \\
\frac{\partial^{2} \dot{U}}{\partial t^{2}}-c^{2}\left[\frac{\partial^{2} U}{\partial x^{2}}+\frac{\partial^{2} U}{\partial y^{2}}\right]=0 \quad \text { and } \\
\frac{\partial U}{\partial t}-\alpha\left[\frac{\partial^{2} U}{\partial x^{2}}+\frac{\partial^{2} U}{\partial y^{2}}\right]=0 .
\end{gathered}
$$

Here $t$ is time, $U$ is the dependent variable, $c$ is the wave velocity, $c_{x}=c \cos (\theta)$ and $c_{y}=c \sin (\theta)$ are the advection velocity components and $\alpha$ is the physical diffusivity. .

The semi-discrete forms of Eq.s (9)-(11) are required for the following analysis. The details for obtaining the Bubnov-Galerkin weak form of these equations are well known [8], and are not repeated here. The semi-discrete. forms of the first-order wave, secondorder wave and parabolic equations are,

$$
\begin{gathered}
M \dot{d}+A(c) d=0, \\
M \ddot{d}+K(c) d=0 \quad \text { and, } \\
M \dot{d}+K(\alpha) d=0,
\end{gathered}
$$

where $A$ is the advection operator, $K$ is the stiffness matrix, and $d$ is the vector of unknown coefficients. In the analysis that follows, the generalized mass matrix is used,

$$
\mathrm{M}=\beta \mathrm{M}^{c}+(1-\beta) \mathrm{M}^{l}
$$

where $\mathbf{M}^{c}$ and $\mathbf{M}^{l}$ are the consistent and row-sum-lumped mass matrices respectively, and $0 \leq \beta \leq 1$ is the lumping parameter.

It should be noted that some form of numerical quadrature is required for the evaluation of $\mathrm{A}, \mathrm{K}$ and $\mathrm{M}$ above. This integration may be performed by placing a grid of quadrature points overlaying the particle points and using Gaussian quadrature. Alternatively, the particle points can be used as the quadrature points with the appropriate weight being the variational volume associated with the particle (trapezoidal or particle integration). Here, both integration techniques and their effects on the discretization errors are considered. 
Proceeding with the Fourier analysis, a plane wave solution is placed on an infinite span (alternatively, on a finite domain with periodic boundary conditions) in order to compare the exact and semi-discrete solutions. It can be shown that the particle values, $\mathrm{U}$, satisfy the same evolution equations as the coefficients, $\mathrm{d}$, when a periodic domain and symmetric window functions are used [25]. Thus, Eq.s (12)-(14) may be rewritten in terms of $U$ for the purposes of this analysis. In this way, the plane wave solution to the hyperbolic Eq.s (9) and (10) may be expressed as,

$$
U(x, y, t)=A \exp [\iota k(x \cos (\theta)+y \sin (\theta))-\iota \omega t],
$$

where $A$ is the amplitude, $\mathrm{k}$ is the wave number, $\omega$ is the circular frequency, $\theta$ is the propagation direction measured from the $\mathrm{x}$-axis, and $\iota=\sqrt{-1}[2,6,25]$.

The solution to the parabolic equation, Eq. (11), is given as

$$
U(x, y, t)=A \exp \left[\iota k(x \cos (\theta)+y \sin (\theta))-\alpha k^{2} t\right] .
$$

\section{Remark}

The use of the term "von Neumann" analysis for the parabolic partial differential equation may be misleading because the equation does not propagate waves as do the hyperbolic equations (although the authors are aware of hyperbolic theory for heat conduction). Instead, the interpretation relies on the fact that while the physical diffusivity is independent of wavelength, the semidiscretization's effective diffusivity is a function of $\lambda$, i.e., $\bar{\alpha}=\bar{\alpha}(\lambda)$. Thus, the analog to dispersion error for the hyperbolic equations is the observation that the wavelength dependent diffusivity leads to errors in the overall rate of diffusion.

Now, consider a domain with particles equally spaced at intervals of $\Delta x$ and $\Delta y$ on a Cartesian grid. Hence, any particle $(i+m, j+n)$ at coordinates $\left(x_{i+m}, y_{j+n}\right)$ may be located relative any other particle $(i, j)$ as $x_{i+m}=x_{i}+m \Delta x$ and $y_{j+n}=y_{j}+n \Delta y$. With the particle spacing defined, the periodic solution to the semi-discrete hyperbolic and parabolic equations are,

$$
\begin{gathered}
U_{i+m, j+n}=U_{i, j} \exp [\iota k(m \Delta x \cos \theta+n \Delta y \sin \theta)-\iota \bar{\omega} t] \quad \text { and } \\
U_{i+m, j+n}=U_{i, j} \exp \left[i k(m \Delta x \cos \theta+n \Delta y \sin \theta)-\bar{\alpha} k^{2} t\right]
\end{gathered}
$$

respectively. Here, $U_{i, j}=A \exp \left[\iota k\left(x_{i} \cos \theta+y_{j} \sin \theta\right)\right]$ and $\bar{\omega}$ and $\bar{\alpha}$ are the effective (numerical) circular frequency and diffusivity respectively.

Given an arbitrarily wide kernel function, the semi-discrete forms of the first- and second-order wave and parabolic equations mäy be rewritten for particle $(i, j)$ as

$$
\sum_{l=-n}^{n} \sum_{m=-n}^{n}\left\{M_{(i, j),(i+l, j+m)} \dot{U}_{i+l, j+m}+A_{(i, j),(i+l, j+m)} U_{i+l, j+m}\right\}=0
$$




$$
\begin{gathered}
\sum_{l=-n}^{n} \sum_{m=-n}^{n}\left[M_{(i, j),(i+l, j+m)} \ddot{U}_{i+l, j+m}+K_{(i, j),(i+l, j+m)} U_{i+l, j+m}\right]=0 \quad \text { and } \\
\sum_{l=-n}^{n} \sum_{m=-n}^{n}\left[M_{(i, j),(i+l, j+m)} \dot{U}_{i+l, j+m}+K_{(i, j),(i+l, j+m)} U_{i+l, j+m}\right]=0,
\end{gathered}
$$

where $M_{(i, j),(i \div l, j+m)}, K_{(i, j),(i+l, j+m)}$ and $A_{(i, j),(i+l, j+m)}$ are the mass, stiffness and advection matrix entries on the row associated with particle $(i, j)$ and the column associated with particle $(i+l, j+m)$. Here, the summation is over the range of all particles under the support of the kernel function at particle $(i, j)$

Computation of the normalized phase and group speed for the semi-discrete hyperbolic equations continues by substituting Eq. (18) and its temporal derivatives into Eq.s (20) and (21) yielding,

$$
\begin{aligned}
& -\iota \bar{\omega} \sum_{l=-n}^{n} \sum_{m=-n}^{n}\left[M_{(i, j),(i+l, j+m)} \exp (\iota k(l \Delta x \cos (\theta)+m \Delta y \sin (\theta)))\right] \\
& +\sum_{l=-n}^{n} \sum_{m=-n}^{n}\left[A_{(i, j),(i+l, j+m)} \exp (\iota k(l \Delta x \cos (\theta)+m \Delta y \sin (\theta)))\right]=0 \\
& -\bar{\omega} \sum_{l=-n}^{n} \sum_{m=-n}^{n}\left[M_{(i, j),(i+l, j+m)} \exp (\iota k(l \Delta x \cos (\theta)+m \Delta y \sin (\theta)))\right] \\
& +\sum_{l=-n}^{n} \sum_{m=-n}^{n}\left[K_{(i, j),(i+l, j+m)} \exp (\iota k(l \Delta x \cos (\theta)+m \Delta y \sin (\theta)))\right]=0
\end{aligned}
$$

for the first- and second-order equations respectively. The normalized phase speed is obtained by rearranging Eq.s (23) and (24) and solving for the effective circular frequency, $\bar{\omega}$. Using the fact that, for a non-dispersive medium, $c=\omega / k$, the normalized phase speed associated with either semi-discrete hyperbolic equation, defined by $\psi=\bar{c} / c$, is

$$
\psi_{1}=\frac{1}{\iota c k} \frac{\sum_{l=-n}^{n} \sum_{m=-n}^{n}\left[A_{(i, j),(i+l, j+m)} \exp (\iota k(l \Delta x \cos (\theta)+m \Delta y \sin (\theta)))\right]}{\sum_{l=-n}^{n} \sum_{m=-n}^{n}\left[M_{(i, j),(i+l, j+m)} \exp (\iota k(l \Delta x \cos (\theta)+m \Delta y \sin (\theta)))\right]}
$$

for the first-order.wave equation and

$$
\psi_{2}=\frac{1}{c k} \sqrt{\frac{\sum_{l=-n}^{n} \sum_{m=-n}^{n}\left[K_{(i, j),(i+l, j+m)} \exp (\iota k(l \Delta x \cos (\theta)+m \Delta y \sin (\theta)))\right]}{\sum_{l=-n}^{n} \sum_{m=-n}^{n}\left[M_{(i, j),(i+l, j+m)} \exp (\iota k(l \Delta x \cos (\theta)+m \Delta y \sin (\theta)))\right]}}
$$

for the second-order wave equation where $\bar{c}$ is the discrete phase speed.

The appropriate error measure that arises for the parabolic PDE is the normalized effective diffusivity, $\bar{\alpha} / \alpha$. Substituting Eq. (19) into (22) yields,

$$
\begin{aligned}
& -\bar{\alpha} k^{2} \sum_{l=-n}^{n} \sum_{m=-n}^{n}\left[M_{(i, j) ;(i+l, j+m)} \exp (\iota k(l \Delta x \cos (\theta)+m \Delta y \sin (\theta)))\right] \\
& +\sum_{l=-n}^{n} \sum_{m=-n}^{n}\left[K_{(i, j),(i+l, j+m)} \exp (\iota k(l \Delta x \cos (\theta)+m \Delta y \sin (\theta)))\right]=0 .
\end{aligned}
$$


Solving Eq. (27) for $\bar{\alpha}$ and dividing through by $\alpha$ yields the desired expression for normalized effective diffusivity,

$$
\bar{\alpha} / \alpha=\frac{1}{\alpha k^{2}} \frac{\sum_{l=-n}^{n} \sum_{m=-n}^{n}\left[K_{(i, j),(i+l . j+m)} \exp (\iota k(l \Delta x \cos (\theta)+m \Delta y \sin (\theta)))\right]}{\sum_{l=-n}^{n} \sum_{m=-n}^{n}\left[M_{(i, j),(i+l, j+m)} \exp (\iota k(l \Delta x \cos (\theta)+m \Delta y \sin (\theta)))\right]} .
$$

The one-dimensional numerical dispersion and diffusion relations may be obtained from the two-dimensional results by setting $\theta=0$. In this way, the phase speed for the first and second-order wave semi-discretizations are,

$$
\begin{gathered}
\psi_{1}=\frac{f_{a}}{c k f_{m}} \\
\psi_{2}=\frac{1}{c k} \sqrt{\frac{f_{k}}{f_{m}}}
\end{gathered}
$$

while the ratio of the effective to physical diffusivity for the parabolic equation is,

$$
\bar{\alpha} / \alpha=\frac{1}{\alpha k^{2}} \frac{f_{k}}{f_{m}}
$$

where

$$
\begin{gathered}
f_{a}=A_{i, i}+2 \sum_{l=1}^{n} \sin (k l \Delta x) A_{i, i+l}, \\
f_{m}=M_{i, i}+2 \sum_{l=1}^{n} \cos (k l \Delta x) M_{i, i+l} \quad \text { and } \\
f_{k}=K_{i, i}+2 \sum_{l=1}^{n} \cos (k l \Delta x) K_{i, i+l} .
\end{gathered}
$$

and a symmetric kernel has been assumed so that the Euler identities (e.g. $2 \cos (z)=$ $\exp (\iota z)+\iota \exp (-\iota z)))$ may be used to simplify the expressions.

In one dimension, the normalized group speed is defined as $\zeta=v_{g} / c$, where $v_{g}=$ $\partial \bar{\omega} / \partial k$. Using Eq. (29) and (30), the normalized group speed in one dimension is

$$
\begin{gathered}
\zeta_{1}=\frac{g_{a} f_{m}-f_{a} g_{m}}{c f_{m}^{2}} \quad \text { and, } \\
\zeta_{2}=\frac{\sqrt{f_{m}}}{2 c \sqrt{f_{k}}} \frac{g_{k} f_{m}-f_{k} g_{m}}{f_{m}^{2}}
\end{gathered}
$$

for the first and second-order wave equations respectively. Here,

$$
g_{a}=\partial f_{a} / \partial k=2 \Delta x \sum_{l=1}^{n} l \cos (k l \Delta x) A_{i, i+l}
$$




$$
\begin{gathered}
g_{m}=\partial f_{m} / \partial k=-2 \Delta x \sum_{l=1}^{n} l \sin (k l \Delta x) M_{i, i+l} \quad \text { and } \\
g_{k}=\partial f_{k} / \partial k=-2 \Delta x \sum_{l=1}^{n} l \sin (k l \Delta x) K_{i, i+l} .
\end{gathered}
$$

Unless otherwise noted, the normalized phase speed and group speed defined above are referred to simply as phase speed and group speed in the remaining text.

Finally, note that there have been no restrictions (with the exception of symmetry) placed on the form or type of shape functions used to obtain the mass, stiffness or advection operators. Thus, Eq. (25) through (36) are valid for Galerkin formulations that use either the RKPM shape functions or the usual finite element shape functions.

\section{Remark}

Note that when the diffusion term is added to the first-order wave (advection) equation (i.e. the linear advection-diffusion equation is considered) the analytic solution (for the case of periodic boundary conditions) is simply the product of those for the pure advection and pure diffusion equations. As a result, the eigenvalue of the advection-diffusion operator is a linear combination of those for pure advection and pure diffusion and thus may be considered seperately, as is done here (see pages 187-188 in [7]).

\subsection{Asymptotic Analysis of von Neumann Results}

In contrast to the von Neumann analysis which provides information concerning the accuracy of the semi-discrete equations over the range of discrete wavelengths, an asymptotic analysis of these results can provide information concerning the rate of convergence of the method in the limit as the particle spacing goes to zero $[6,7,25,26]$. As an example, consider the truncation error of a one-dimensional semi-discretization of the advection equation,

$$
T^{h}=K \Delta x^{q}\left(\frac{\partial^{q+1} U}{\partial x^{q+1}}\right)+\text { higher order terms }=O\left(\Delta x^{q}\right)
$$

where $K$ is a constant independent of $U$ and $\Delta x$, and $q$ is the asymptotic rate of convergence of the semi-discretization. Vichnevetsky, et al. have shown that there is a direct relationship between rate of convergence, $q$, of Eq. (40) and "flatness" of the discrete phase speed, $\bar{c}(k)$, as $k \rightarrow 0$ [25]. Indeed, they have shown that

$$
\bar{c} / c-1=\psi-1=K \Delta x^{q}(\iota k)^{q} / c+\text { higher order terms }=O\left(k^{q}\right) .
$$

where $q$ in Eq. (41) is identical to that in Eq. (40). Hence, to evaluate $q$, a Taylor series expansion of $\psi$ is taken about $k=0$ yielding,

$$
\psi-1=\left[\psi(k=0)+k\left[\frac{\partial \psi}{\partial k}\right]_{k=0}+\frac{k^{2}}{2 !}\left[\frac{\partial^{2} \psi}{\partial k^{2}}\right]_{k=0}+\cdots\right]-1
$$


The lowest order non-zero term in Eq. (42) sets the rate of convergence of the semidiscretization. In a similar manner, the asymptotic behavior of the second-order wave equation may be obtained. The rate of convergence for the parabolic model equation follows similarly, with the expansion

$$
\bar{\alpha} / \alpha-1=\left[\bar{\alpha}(k=0)+k\left[\frac{\partial \bar{\alpha}}{\partial k}\right]_{k=0}+\frac{k^{2}}{2 !}\left[\frac{\partial^{2} \bar{\alpha}}{\partial k^{2}}\right]_{k=0}+\cdots\right] / \alpha-1
$$

used to evaluate $q$. Again, the lowest order non-zero term sets the asymptotic rate of convergence.

Calculation of these asymptotic rates, while straight-forward, involves considerable algebraic manipulation. Further, each choice of refinement parameter and mass matrix formulation results in a unique asymptotic expression. In order to conserve space, only the resulting asymptotic rates of convergence are presented here. The interested reader is directed to the literature for example calculations [25].

\section{Results}

This section summarizes the RKPM von Neumann and asymptotic analyses results. These results were obtained using the cubic spline kernel function of Eq. (3) with linear reproducing conditions enforced as outlined in $\S 2.1$. The effect of integration on discretization errors is investigated by considering both fully integrated and pointwise (or trapezoidally) integrated advection, stiffness and mass matrices. Because a closed form of the RKPM shape functions is not generally available, full integration is achieved by employing Gaussian quadrature with 4 quadrature points between each pair of particles. Doubling the number of quadrature points was found to have a negligible impact on the effective diffusivity or phase speed for the semi-discretizations considered.

As noted in $\S 2.1$, the width (or support) of the kernel function, $\bar{\varphi}$, may be controlled by adjusting the refinement parameter, $r$. In this way, the number of particles under the function is controlled. Since the ability to refine the support (and hence resolve finer scales of the modeled physics) is an important attribute of RKPM, the effect of $r$ is of concern. For this reason, a range of refinement parameters is considered. In particular, refinement parameters corresponding to kernel functions of 3 and 5 particle support ( $r=0.5$ and 1.0 respectively) are investigated. The transition from 3 to 5 particle support is also examined with $r=0.75$ (symmetric kernel functions cover odd numbers of particles so that 4 particle support is not possible). Finally, the energy error minimizing refinement parameter (EEMIR) derived by Liu and Chen is used. For the cubic spline kernel functions used here, the EEMR is $r=1.14$ [13].

In the discussion that follows, the dispersion, phase and group speed' 'results:are presented as functions of non-dimensional wave number, $2 \Delta x / \lambda=k \Delta x / \pi$. In order to simplify the discussion, the following nomenclature has been adopted to identify the mass matrix and quadrature rule used. The mass matrix is identified as $C$ for consistent 
$(\beta=1), L$ for row-sum lumped $(\beta=0)$, or $H$ for higher-order $(\beta=1 / 2)$; cf. Eq. (15). The numerical integration scheme is identified as either $F$ for full integration (as defined above) or $T$ indicating a trapezoidal rule (i.e. particle integration).

For the purpose of comparison, results are presented for linear FEM semi-discretizations. The concomitant effective diffusivity, phase speed and group speed are calculated using the formulae presented in $\S 2.2$ with linear finite element shape functions. The linear FEM basis was chosen for comparison as it provides the same degree of consistency as the RKPM discretizations used here.

\subsection{First-Order Wave Equation}

Normalized numerical phase speed is presented in Figure 1 for several FEM and RKPM semi-discretization techniques and a range of refinement parameters. Results are plotted for fully integrated consistent (CF), trapezoidally-integrated consistent (CT), fully integrated higher-order (HF) and fully integrated lumped (LF) mass formulations. For the purposes of interpretation, an ideal semi-discretization (in terms of phase speed) would yield $\psi=1$ for all resolvable wavelengths (i.e., $2 \Delta x / \lambda \leq 1$ ).

Figure 1(a) shows the (strictly lagging) phase speed for the $C F$ formulation. It is clear from the figure that the EEMR, $r=1.14$, provides the best phase response. As $r$ is decreased from its EEMR value, the phase speed deteriorates, achieving the limiting case of the linear FEM. Figure 1 also illustrates the identical phase speed of the linear FEM and the $r=0.5$ RKPM methodologies. This results as linear FElI and RKPM $r=0.5$ shape functions are the same for uniform particle spacing. All CF discretizations yield $\psi_{1}=0$ at the Nyquist limit of the grid, indicating that the $2 \Delta x$ waves are stationary (the energy of these waves travels at their non-zero group velocity; cf. Figure 2). It is noted that the $C F, r=1.0$ phase speed results are identical to those of Vichnevetsky and Bowles [25] where the cubic B-spline was used in a Galerkin weak form solution of the first-order wave equation. Indeed, this is to be expected as the cubic spline kernel function, $\varphi$, used here is identically the cubic B-spline when $r=1$ and the domain is periodic. As the cubic B-spline automatically meets the moment conditions of $\S 2.1$ for all moments, $\varphi$ and $\bar{\varphi}$ are the same and the RKPM and Vichnevetsky test functions are identical (and hence yield identical semi-discretizations). When other $r$ are used, $\bar{\varphi}$ is no longer the true cubic B-spline and the RKPM results differ from those of Vichnevetsky. Indeed, the contribution of RKPM is to allow variable $r$ while retaining convergence of the method (cf. Table 1).

Particle integration of the consistent mass and stiffness matrices (CT) yields a semidiscretization which produces lagging phase speed over the range of resolvable wavelengths (cf. Figure $1(\mathrm{~b})$ ). As with the $\mathrm{CF}$ results, an increasing refinement parameter corresponds to improved phase speed although the CF methodology outperforms the CT discretization for all $r$ considered. As with the CF formulation, CT phase speed goes to zero at the Nyquist limit of the grid.

The effect of mass lumping on phase speed is demonstrated in Figure 1 (d). The 
LF formulation provides substantially worse phase speed over most of the resolvable wavelengths, matching $\mathrm{CF}$ and $\mathrm{CT}$ methodologies only at $2 \Delta x / \lambda=0,1$. Unlike the consistent mass matrix formulations, increasing $r$ for the lumped mass semi-discretization increases the lagging phase errors. A comparison of Figures 1(c) and (d) show the increased complexity of the higher order mass formulation only slightly improves the phase speed results relative to the LF formulation.

Normalized group speed corresponding to the semi-discretizations of Figure 1 are presented in Figure 2. For the purposes of comparison, $v_{g}=c$ and thus $\zeta_{1}=1$ in the non-dispersive medium represented by the continuous form of the first-order wave equation.

Figure 2(a) shows the group speed associated with the CF formulation. The figure indicates that increased $r$ results in greater accuracy in the sense that $\zeta_{1}$ remains close to 1 at shorter wavelengths. However, increasing $r$ also results in a substantial increase in the magnitude of the maximum (negative) group velocities that occur at $2 \Delta x$. Gresho [7] has noted that first-order wave equation semi-discretizations which accurately represent phase speed for shorter wavelengths produce greater negative group velocities for the shortest wavelengths they cannot represent. These negative group speeds increase as $\partial \psi_{1} / \partial k$ must increase for $\psi_{1}$ to transition from 1 to 0 over shorter $2 \Delta x / \lambda$ (presuming $\psi_{1}(\lambda=2 \Delta x)=0$ for semi-discretizations of the first-order wave equation). Thus, the appearance of large negative group velocities suggests a method with good phase speed characteristics. It will be demonstrated in $\S 3.2$ that the connection between large negative group velocities and good phase behavior is not true for semi-discretizations of the second-order wave equation.

The effect of point-wise integration on group speed is evident in a comparison of the CF and CT results (cf. Figures 2(a)-(b)). Both methods show increasing accuracy in terms of group speed as $r$ is increased. Relative to the CF methodology, the CT methodology exhibits substantially reduced maximum negative group speed at $\lambda=2 \Delta x$, reflecting the poorer phase speed behavior of the CT method.

As with the phase speed, lumping of the mass matrix has a dramatic effect on group speed, further reducing their maximum negative values, again, reflective of their poor phase speed. The higher order mass matrix has only a minor influence on group speed relative to the LF formulation. Surprisingly, the maximum negative group speed, which occurs at $\lambda=2 \Delta x$ for the CF and CT semi-discretizations, has shifted to longer wavelengths for the LF and HF schemes characterized by $r=1.0$ and 1.14. Maximum negative group speed for the $r=0.5$ and $0.75 \mathrm{LF}$ and HF formulations occur at $\lambda=2 \Delta x$.

In order to quantify the performance of the methods considered, an error measure (the phase $\gamma$-accuracy [26]) that defines the length of the segment along the $2 \Delta x / \lambda$ axis $\because \because$ between zero and the point $(2 \Delta x / \lambda)_{\gamma}$ beyond which $\epsilon_{\psi}=|\psi-1| \geq \gamma$, is employed. For engineering purposes $\gamma=0.05$ is assumed to be appropriate.. Table 1 shows the minimum number of particles required per wavelength, $N_{\gamma}$, to satisfy the stated criteria where $N_{\gamma}$ has been rounded up to the nearest integer. Again, it is clear that RKPMI with $r=1.14$ outperforms the linear FEM formulation, requiring only 4 particles per 
wavelength in contrast to the 5 nodes required by FEM. Table 1 also indicates that for $r=1.14$, the CT semi-discretization performs as well as the CF methodology in the $N_{\gamma}$ sense. Since the CT method requires fewer computations and no background quadrature grid as does its CF counterpart, these results suggest that the CT method may be a practical alternative to the CF method. Finally, as suggested by Figure 1, the LF and HF methods result in a significant deterioration in phase speed relative to the consistent mass methods, requiring substantially more particles per wavelength to produce the same phase $\gamma$-accuracy. Indeed, the "best" LF ( $r=0.5-$ FEM) and "worst" CT $(r=0.5)$ formulations require 13 and 6 particles per wavelength respectively.

An error measure similar to the phase $\gamma$-accuracy can be defined for the group speed. Specifically, the group $\gamma$-accuracy defines the length of the segment along the $2 \Delta x / \lambda$ axis between zero and the point $(2 \Delta x / \lambda)_{\gamma}$ beyond which $\epsilon_{\zeta}=|\zeta-1| \geq \gamma$. As with the phase accuracy, $\gamma$ is taken to be 0.05 . Table 1 shows the minimum number of particles required per wavelength, $N_{\gamma}$, to satisfy this group error criteria. Again, the consistent mass matrix formulations display the best performance with the CF and CT $r=1.14$ methods requiring 4 and 5 particles per wavelength respectively. A comparison of the phase and group values of $N_{\gamma}$ indicate that when $\epsilon_{\psi}=\epsilon_{\zeta}$ the group $\gamma$-accuracy criteria is more restrictive than that for phase. As with the phase $\gamma$-accuracy, group $\gamma$-accuracy indicates that the LF and HF formulations are quite inferior to the consistent mass formulations. For example, the "worst" consistent mass formulation (CT, $r=0 . \overline{5}$ ) requires 7 particles per wavelength, while the "best" LF method needs, at a minimum, 21 particles.

Results of the asymptotic analysis described in $\S 2.3$ are shown in Table 2. It is clear from the table that excellent asymptotic rates of convergence are possible with the appropriate choice of refinement parameter. Conversely, a poor choice of $r$ may reduce this asymptotic rate; although rates are still comparable to those for the linear FEM. It should be noted that although the rates of convergence for the RKPM methods are generally comparable to those for FEM, this is only a measure of the optimal rate of convergence of the method (quality of the approximation as $\lambda \rightarrow \infty$ ). In fact, solutions are comprised of short to long wavelength components, all of which contribute to the error of the semi-discretization [26]. Thus, as indicated by Gresho [7]. the effective diffusivity, phase and group speeds tell much more of the story and rates of convergence should be interpreted carefully.

\subsection{Second-Order Wave Equation}

Results for FEM and RKPM CF, CT, HF and LF semi-discretizations are plotted in Figure 3. As with the first-order wave equation, an ideal semi-discretization (in terms of phase speed) would yield $\psi_{2}=1$ for all resolvable wavelengths (i.e., $2 \Delta x / \lambda \leq 1$ ). Figure 3(a) shows the strictly leading phase speed results for the CF formulation. Clearly the best phase speed is achieved with the EEMR, $r=1.14$. Incredibly, nearly perfect phase speed is obtained for the range of $2 \Delta x / \lambda$ when this refinement parameter is used. 
Decreasing $r$ below 1.14 results in degraded phase speeds with the worst behavior demonstrated by the linear FEM (RKPM $r=0.5$ ) methodology. All formulations considered show strictly leading phase speeds. Irregardless of the value of $r$, zero phase speed is never achieved for the range of resolvable wavelengths, indicating that no wavelengths are stationary on the grid.

The effect of point-wise integration of the consistent mass formulation (CT) is illustrated in Figure 3(b). Again, increasing $r$ results in reduced lagging phase errors. Relative to the CF methodology, the CT method results in increased lagging phase errors for all but very long wavelength waves $(2 \Delta x / \lambda \approx 1)$. Indeed, the general behavior of $\psi_{2}$ has changed dramatically relative to the $\mathrm{CF}$ formulation with phase speeds lagging over the range of wavelengths, going to zero at $\lambda=2 \Delta x$.

Interestingly, the use of trapezoidal integration (with a consistent mass matrix) results in identical dispersion relations for the first and second-order wave equations (cf. Figures 1(b) and 3(b)). Similar behavior has been noted by Vichnevetsky and Bowles [25] when a second-order central difference approximation is applied to both the first and second-order wave equation. In this situation, the semi-discrete first-order equation is a consistent representation of both the first-order wave and the second-order wave equation for the right-moving wave. In the case of RKPM, a similar result has been obtained for $r=0 . \bar{j}$. However, we have been unable to verify this behavior analytically for the other refinement parameters considered here. Regardless, numerical experiments (cf. Figures 1 and 3 ) have verified that the discrete spectrum is identical for the two model hyperbolic equations when trapezoidal integration is used.

Figures 3(c) and (d) show the numerical phase speed results for the HF and LF formulations respectively. Both methodologies exhibit lagging phase speeds for the entire range of discrete wavelengths. At the Nyquist limit, phase speed is non-zero for all $r$ and depends on the choice of $r$. Thus, unlike the CT formulation, all waves propagate at non-zero speed. As with the HF and LF formulations of the first-order wave equation, lagging phase errors increase with increasing $r$ for any dimensionless wavelength. Again, the $\mathrm{HF}$ formulation provides little improvement over the LF formulation.

Normalized group speed corresponding to the semi-discretizations of Figure 3 are presented in Figure 4. The group speed associated with the CF formulation indicates increased accuracy corresponding to increased $r$ (cf. Figure 4(a)). Indeed, RKPM with $r=1.14$ performs substantially better than linear FEM, producing nearly perfect group speed to $2 \Delta x / \lambda=0.7$. Surprisingly, group velocities are positive for the entire range of discrete wavelengths with $\zeta_{2}$ going to zero at $2 \Delta x / \lambda=1$. Thus, energy associated with all but $2 \Delta x$ waves (which remain stationary) travels in the same direction as the waves themselves.

The effect of particle integration on the consistent mass semi-discretization of the second-order wave equation is demonstrated in Figure 4(b). A significant effect of particle integration seems to be the introduction of negative group relocities which reach a maximum at $\lambda=2 \Delta x$. It is clear from Figure 3(a)-(b) that the CF method produces better phase speed than does the CT formulation. Thus, unlike semi-discretizations of 
the first-order wave equation, negative group velocity associated with the second-order wave formulations does not suggest good phase behavior.

Figures 4(c) and (d) show the numerical group speed results for the HF and LF formulations respectively. As with the CT formulation, the HF and LF systems can produce negative group speed. Surprisingly, however, negative group speed is achieved only for $r=1$ and 1.14 semi-discretizations (the maximum value is less than for the CT method). When the $r=0.5$ and 0.75 semi-discretizations are employed, the group speeds are strictly positive. Further, as with the first-order HF and LF semi-discretizations corresponding to $r=1.0$ and 1.14, the group speed reaches a (negative) maximum at wavelengths less than the Nyquist limit.

As for the first-order wave equation: phase $\gamma$-accuracy is defined with $\gamma=0.05$ assumed appropriate for engineering purposes. These results are presented in Table 3 for the $\mathrm{CF}, \mathrm{CT}, \mathrm{HF}$ and LF formulations already considered. Again, it is clear that CFRKPM with $r=1.14$ outperforms all other formulations investigated here, requiring 3 particles (the Nyquist sampling limit) per wavelength. As a comparison, linear CF-FEMI requires 7 particles per wavelength while LF-RKPM $(r=1.14)$ requires 14 particles. The best linear FEM formulation (HF) requires 4 nodes per wavelength although it results in substantially worse phase speed relative to CF-RKPM beyond this limit (cf. Figure 3). Additionally, the CT formulation performs nearly as well as the $\mathrm{CF}$ formulation for $r=1.14$ in terms of $N_{\gamma}$ although, as with HF-FEM, its phase speed behavior beyond this point is not comparable to that of the best CF-RKPM.

Although the consistent mass methodologies may produce substantially better phase speed than the LF or HF formulations when $r$ is chosen appropriately $(r \geq 1.0)$ poor choice of $r$ can degrade their performance. Indeed, the LF and HF methods may perform as good as or even slightly better than consistent formulations provided $r$ is not chosen wisely (cf. Table 3 ). For example, HF with $r=0.5$ requires only 4 particles per wavelength while $C F$ with the same $r$ requires 7 particles for the same accuracy. Clearly, the choice of the refinement parameter and mass matrix formulation are important to the performance of these methods.

Results of the group $\gamma$-accuracy (defined similar to that of the first-order discretizations) are also presented in Table 3. Clearly, the group $\gamma$-accuracy is more restrictive than is the phase $\gamma$-accuracy. Again, the CF-RKPM method with $r=1.14$ performs the best requiring 4 particles per wavelength while the best linear FEM methodology (HF) requires 6 nodes. The $\mathrm{CT}$ formulation performs nearly as well as the $\mathrm{CF}$ formulation for the EEMR although, again, demonstrates negative group velocity not evident for the CF method. The worst performance is demonstrated by the LF-RKPM method with $r=1.14$.

Asymptotic analysis results are shown in Table 4. As with the first-order wave equation excellent asymptotic rates of convergence are possible with the appropriate choice of refinement parameter. Conversely, values of $r$ may be chosen which reduce these rates to those comparable to linear FEM. Again, it is emphasized that the asymptotic analysis provides information concerning the behavior of the discretization around $k=0$. 
Information concerning the global accuracy of the method requires examination of the behavior of the semi-discretization over the range of discrete wavelengths.

\subsubsection{Parabolic Equation}

The normalized effective diffusivity results for the parabolic partial differential equation are presented in Figure 5 for several FEM and RKPM semi-discretizations (CF, CT, $\mathrm{HF}$ and LF) and a range of refinement parameters. Recall that the physical diffusivity is independent of wavenumber, thus $\bar{\alpha} / \alpha=1$ over all discrete wavelengths for an ideal semi-discretization. With this in mind, the CF formulation (Figure 5(a)) with the EEMR, $r=1.14$, performs best though slightly over-diffusive for short wavelengths (maximum $\bar{\alpha} / \alpha=1.06)$. As $r$ is decreased from 1.14 , the method becomes increasingly over-diffusive. The worst performance is exhibited by the linear FEMI (or RKP.II with $r=0.5$ ) semidiscretization with maximum $\bar{\alpha} / \alpha=1.4 \bar{j}$ at $2 \Delta x / \lambda=0.8$.

Particle integration of the mass and stiffness matrices (CT) yields semi-discretizations that are under-diffusive for all values of $r$ investigated, although the point at which $\bar{\alpha} / \alpha$ diverges substantially from 1 (relative to our $5 \%$ error criteria) increases with increased refinement parameter. Another important point is that for $\lambda=2 \Delta x, \bar{\alpha}$ is zero. This indicates that $2 \Delta x$ waves will persist (i.e. they will not be damped) resulting in a "noisy" solution. The fully integrated methodologies investigated here retain some (small for LF and $\mathrm{HF}$ with large $r$ ) diffusive character at these wavelengths.

The effect of mass lumping on effective diffusivity is demonstrated in Figure 5(d). Unlike the consistent mass formulations, increasing $r$ in the lumped mass semi-discretization has the effect of destroying the accuracy of the solution in terms of effective diffusivity at high wavenumbers. As is clear from a comparison of Figures 5(c)-(d), the added complexity of the higher order mass formulation improves, slightly, the diffüisive character relative to the LF.

For concreteness, we consider the $\gamma$-accuracy results of Table 5 . Again, the best performers are the consistent mass formulations with $r$ set to the EEMR. For all $r$ the CF methodology is clearly more accurate than the CT formulations. While the HF FEM formulation performs nearly as well as the best CT methodology, increased $r$ rapidly destroys the method. Finally, as decreasing $r$ has the effect of decreasing the accuracy of the CF and CT methods while increasing that of the LF and HF methods, the latter methods do perform better than the former at small $r$. For example LF outperforms CT for $r=0.75$. Of course both these formulations are much inferior to CF (or even CT) with $r=1.14$.

As with the hyperbolic equations, the refinement parameter modifies the results of the asymptotic analysis (cf. Table 6).: The best convergence rates $\left(O\left(\Delta x^{6}\right)\right)$ possible are for the consistent mass matrix formulation with $r=1$ where the cubic B-spline is obtained. Convergence rates comparable to those for linear FEMI are obtained for the other semi-discretizations considered. 


\section{Conclusions}

Numerical errors associated with the RKPM discretization of the parabolic, first-order and second-order wave equations have been evaluated here in terms of von Neumann analysis. The semi-discretizations considered enforced linear consistency and employed variations on matrix integration (full Gauss quadrature or particle integrations) and type (consistent, lumped or higher order). All formulations incorporate a cubic spline kernel function with the EEMR, $r=1.14$. Linear FEM semi-discretization results are also presented for the purpose of comparison. In light of these analyses the following conclusions may be drawn.

Irregardless of the partial differential equation considered, the consistent mass, fully integrated RKPM formulation employing the EEMR produces the best numerical performance. In terms of the hyperbolic equations, only 3 to 4 particles per wavelength are required to meet a $5 \%$ phase error criteria while linear FENI requires 4 to 5 nodes. Similarly, the parabolic equation requires only 4 particles per warelength to satisfy a $5 \%$ diffusivity error criteria in contrast to linear FEMI which requires 10 nodes for the consistent mass semi-discretization. Incredibly, RKPM semi-discretizations of the second-order wave equation require only 3 particles per wavelength (the Nyquist limit) for phase errors of less than 2.j percent

Results indicate that point-wise or trapezoidal integration results in only a minor degradation in phase speed for the first-order wave equation when the EEMR is used. This suggests that the CT methodology may be an attractive alternative to Gaussian quadrature which is computationally intensive and requires a background integration grid. The application of trapezoidal integration to the second-order wave and parabolic equations is more problematic. Although the number of particles needed to achieve the desired accuracy in terms of phase speed or diffusivity is only slightly increased for CT relative to CF semi-discretizations, the behavior of the phase (first-order wave equation) or diffusivity (parabolic equation) is substantially degraded for the CT formulation. Indeed, the nearly perfect phase speed of the $C F$ second-order ware semi-discretizations is destroyed when trapezoidal integration is used. Further, the CT semi-discretization introduces negative group velocity that is not evident in the CF formulation.

Finally, the lumped and higher order mass formulations introduce severely lagging phase speed for the hyperbolic equations and over-diffusive behavior for the parabolic equation. Indeed, the consistent mass matrix formulations significantly outperform the lumped and higher order formulations for all but small $r(r=0.5$ and $r \leq 0.75$ - lumped and higher order matrices respectively). It should be noted that although the consistent mass formulations can substantially outperform the lumped formulations, poor choice of $r$ (i.e. $r \leq 0.75$ ) produce formulations which are actually worse than the lumped and higher order approximations in terms of phase speed and diffusirity accuracy. 


\section{References}

[1] T. Belytschko, Y. Krongauz, D. Organ, M. Fleming, and P. Krysl, Meshless methods: An overview and recent developments, Computer Methods in Applied Mechanics and Engineering, 139 (1996), pp. 3-47.

[2] T. Belytschko AND R. Mullen, On dispersive properties of finite element solutions, in Modern Problems in Elastic Wave Propagation, J. Milovitz and J. D. Achenbach, eds., International Union of Theoretical and Applied Mechanics, John Wiley and Sons, 1978, pp. 67-82.

[3] J.-S. Chen, S. Yoon, H.-P. WANG, AND W. K. LIU, An improved reproducing kernel particle method for nearly incompressible hyperelastic solids, Computer Methods in Applied Mechanics and Engineering, (submitted 1997).

[4] Y. ChEN, R. A. URAS, AND W. K. LIU, Enrichment of the finite element method with reproducing kernel particle method, in Joint ASNE/JSME pressure vessels and piping conference, ASME/JSME, July 1995.

[5] M. A. Christon AND T. E. Voth, Results of von Neumann analyses for reproducing kernel semi-discretizations, International Journal for Numerical Methods in Engineering, to appear (1998). (Invited paper from the Fourth World Congress on Computational Mechanics).

[6] P. M. Gresho, S. T. Chan, R. L. Lee, and C. D. Upson, A modified finite element method for solving the time-dependent navier-stokes equations. part 1: Theory, International Journal for Numerical Methods in Fluids, 4 (1984); pp. 557-598.

[7] P. M. Gresho and R. L. SAni, Incompressible Flow and the Finite Element Method: Advection and Isothermal Laminar Flow, John Wiley and Sons, Chichester, England, 1998.

[8] T. J. R. Hughes, The Finite Element Method, Linear Static and Dynamic Finite Element Analysis, Prentice-Hall, Englewood Cliffs, New Jersey, 1987.

[9] S. Jun, W. K. LIU, AND T. BELYTSCHKO, Explicit reproducing kernel particle methods for large deformation problems, International Journal for Numerical Methods in Engineering, (1997).

[10] S. LI AND W. K. LIU, Moving least square reproducing kernel method part $i$ : Fourier analysis, 'Computer Methods in Applied Mechanics and Engineering, 139 (1996), pp: 159-193.

[11] — Synchronized reproducing kernel interpolant via multiple wavelet expansion, Computational Mechanics, (Accepted for publication March, 1997). 
[12] —-, Moving least square reproducing kernel method (iii): Wavelet packet and its applications, Computer Methods in Applied Mechanics, (submitted April, 1997).

[13] W. K. LIU AND Y. Chen, Wavelet and multiple scale reproducing kernel methods, International Journal for Numerical Methods in Fluids, 21 (1995), pp. 901-931.

[14] W. K. Liv, Y. Chen, C. T. Chang, and T. BelytschKo, Advances in multiple scale kernel particle methods, Computational Mechanics, 18 (1996), pp. 73-111.

[15] W. K. Liu, Y. Chen, J. S. Chen, T. Belytschko, C. Pan, R. A. Curas, AND C. T. CHANG, Overview and applications of the reproducing kernel particle methods, Archives of Computational Methods in Engineering; 3 (1996), pp. 3-80.

[16] W. K. Liu, Y. Chen, R. A. Uras, and C. T. Chang, Generalized multiple scale reproducing kernel particle methods, Computer Methods in Applied Mechanics and Engineering, 139 (1996), pp. 91-157.

[17] W. K. LIU AND S. JUN, Multiple scale reproducing kernel particle methods for large deformation problems, International Journal for Numerical Methods in Engineering, (submitted 1997).

[18] W. K. Liu, S. Jun, S. Li, J. Adee, And T. Belytschko, Reproducing kernel particle methods for structural dynamics, International Journal for Numerical Methods in Engineering, 38 (1995), pp. 1655-1679.

[19] W. K. LIU, S. Jun, AND Y. F. ZHANG, Reproducing kernel particle methods, International Journal for Numerical Methods in Fluids, 20 (1995), pp. 1081-1106.

[20] W. K. LIU, S. LI, AND T. BELYTSChKo, Moving least square reproducing kernel methods (i) methodology and convergence, Computer Methods in Applied Mechanics and Engineering, (Accepted for publication - June, 1996).

[21] W. K. LiU And C. Oberste-Brandenburg, Reproducing kernel and wavelet particle methods, in Aerospace Structures: Nonlinear dynamics and system response, J. P. Cusumano, C. Peirre, and S. T. Wu, eds., New York, New York, November 1993, ASME Winter Annual Meeting, American Society of Mechanical Engineers, pp. $39-55$.

[22] W. K. LIU, R. A. URAS, AND Y. CHEN, Enrichment of the finite element method with the reproducing kernel particle method, Journal of Applied Mechanics, (accepted for publication, 1997).

[23] R. A. Uras, C. T. Chang, Y. ChEN, and W. K. LiU, Multiresolution réproducing kernel particle methods in acoustics, Journal of Computational Acoustics, (Accepted for publication, 1996). 
[24] R. VICHNEVETSKY, Wave propagation analysis of difference schemes for hyperbolic equations: a review, International Journal for Numerical Methods in Fluids, 7 (1987), pp. 409-452.

[25] R. Vichnevetsky and J. B. Bowles, Fourier Analysis of Numerical Approximations of Hyperbolic Equations, SIAM, Philadelphia, PA, 1982.

[26] R. VICHNEVETSKY AND F. D. SCHUTTER, A frequency analysis of finite difference and finite element methods for initial value problems, in AICA International Symposium on Computer Methods for Partial Differential Equations, Lehigh University, Bethlehem, Pennsylvania, June 1975, pp. 179-185. 

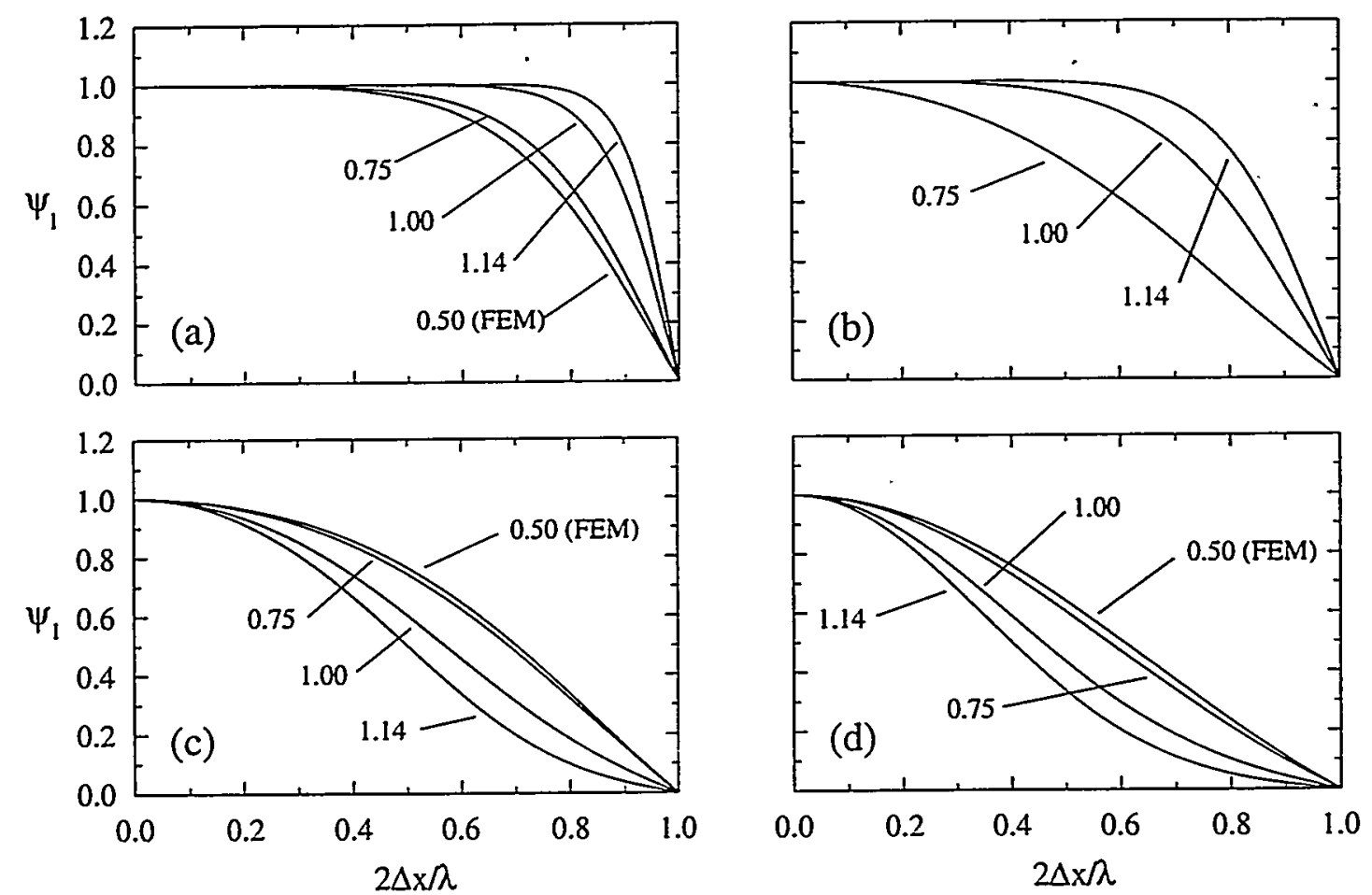

Figure 1: First-order wave equation phase speed for the (a) fully integrated consistent mass (CF), (b) trapezoidally integrated consistent mass (CT), (c) fully integrated higher order mass (HF) and (d) fully integrated lumped mass (LF) RKP.I semi-discretizations. Results are presented for a range of refinement parameters. 

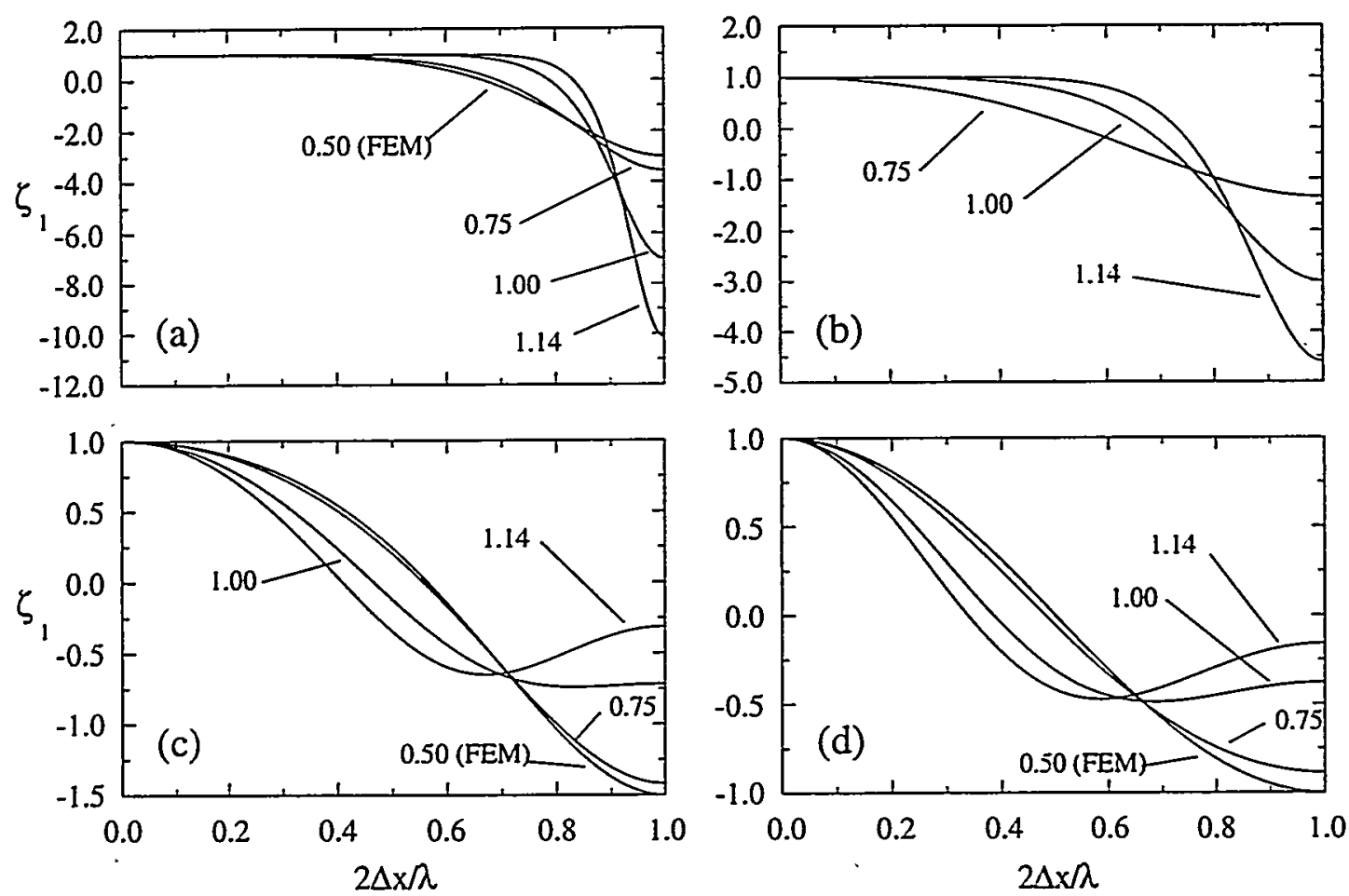

Figure 2: First-order wave equation group speed for the (a) fully integrated consistent mass $(\mathrm{CF}),(\mathrm{b})$ trapezoidally integrated consistent mass $(\mathrm{CT}),(\mathrm{c})$ fully integrated higher order mass (HF), and (d) fully integrated lumped mass (LF) RKPM semi-discretizations. Results are presented for a range of refinement parameters. 

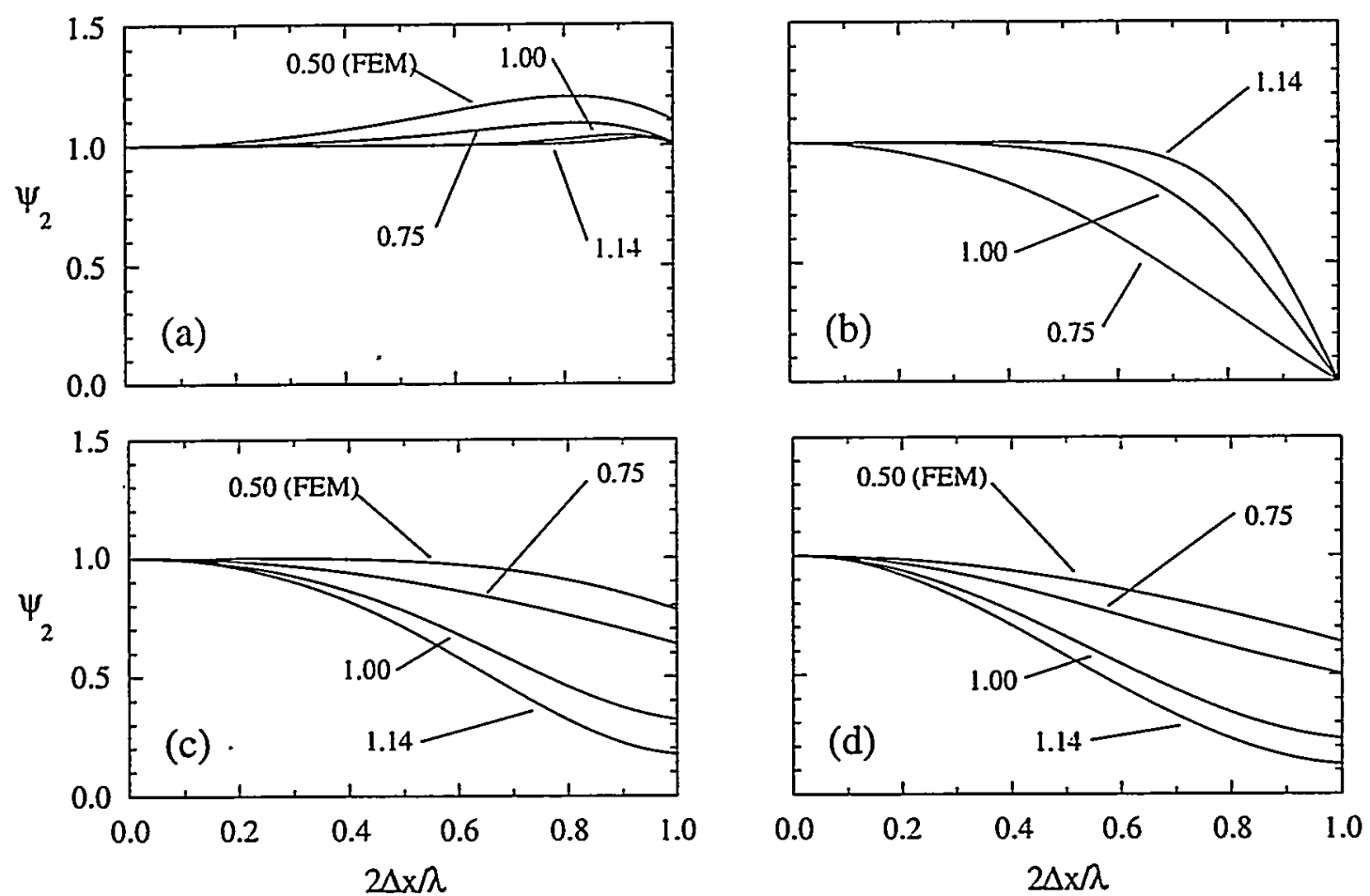

Figure 3: Second-order wave equation phase speed for the (a) fully integrated consistent mass (CF), (b) trapezoidally integrated consistent mass (CT), (c) fully integrated higher order mass (HF), and (d) fully integrated lumped mass (LF) RKPM semi-discretizations. Results are presented for a range of refinement parameters. 

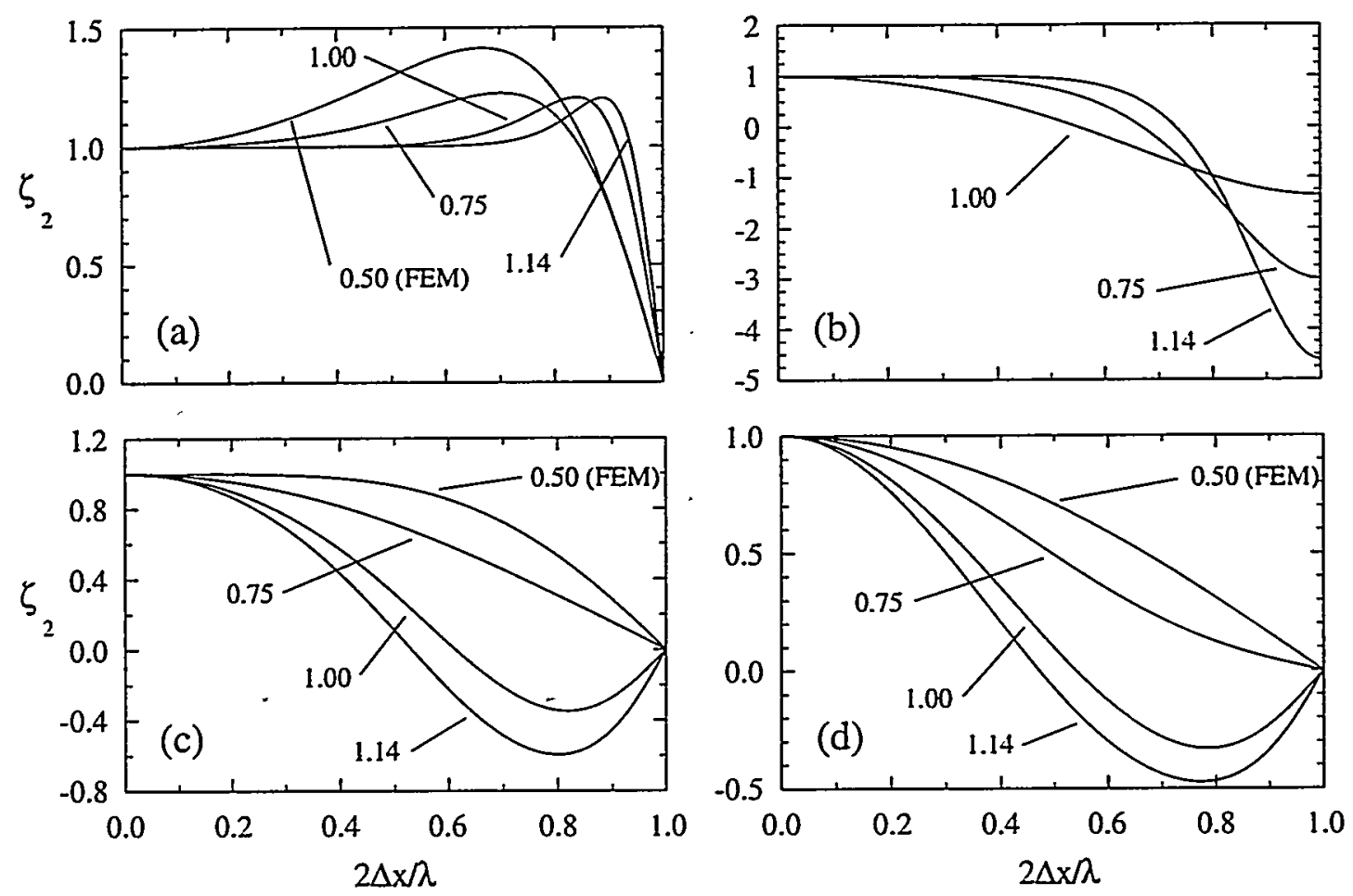

Figure 4: Second-order wave equation group speed for the (a) fully integrated consistent mass (CF), (b) trapezoidally integrated consistent mass (CT), (c) fully integrated higher order mass (HF), and (d) fully integrated lumped mass (LF) RKPM semi-discretizations. Results are presented for a range of refinement parameters. 

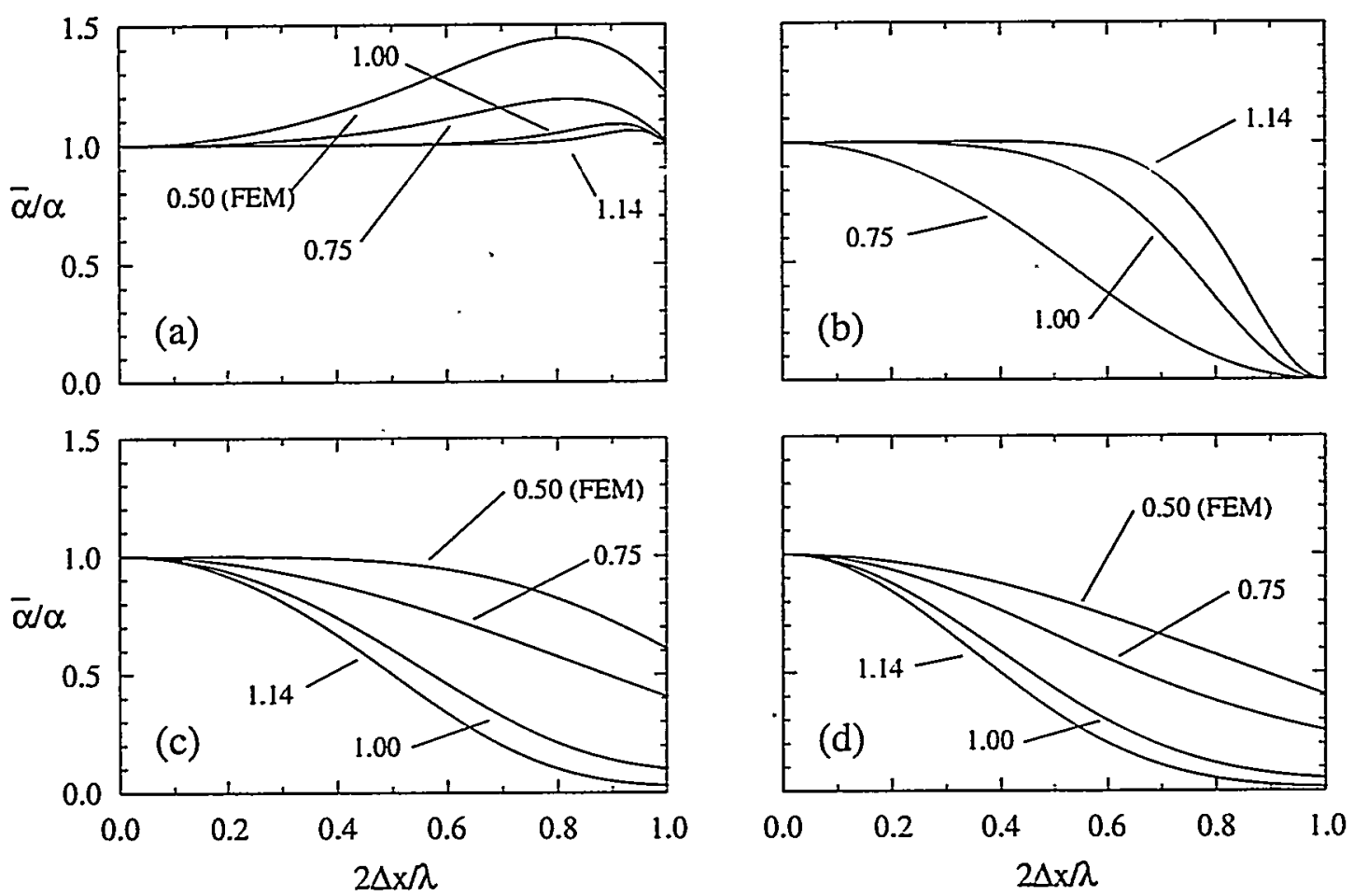

Figure 5: Parabolic PDE numerical diffusivity for the (a) fully integrated consistent mass (CF), (b) trapezoidally integrated consistent mass (CT), (c) fully integrated higher order mass (HF), and (d) fully integrated lumped mass (LF) RKPMI semi-discretizations. Results are presented for a range of refinement parameters. 


\begin{tabular}{|c|c|c|c|c|c|}
\hline \multirow[b]{2}{*}{ Wethod } & \multirow[b]{2}{*}{$r$} & \multicolumn{2}{|c|}{ Phase } & \multicolumn{2}{|c|}{ Group } \\
\hline & & $(2 \Delta x / \lambda)_{\gamma}$ & $N_{\gamma}$ & $\overline{(2 \Delta x / \lambda)_{\gamma}}$ & $N_{\gamma}$ \\
\hline \multirow[t]{4}{*}{$\mathrm{CF}$} & 0.50 (FEM) & 0.51 & 5 & 0.35 & 7 \\
\hline & 0.75 & 0.56 & 5 & 0.40 & 6 \\
\hline & 1.00 & 0.76 & 4 & 0.61 & 5 \\
\hline & 1.14 (EEMR) & 0.83 & 4 & 0.70 & 4 \\
\hline \multirow[t]{4}{*}{ CT } & 0.50 (FEM) & N/A & N/A & N/A & $\mathrm{N} / \mathrm{A}$ \\
\hline & 0.75 & 0.22 & 11 & 0.13 & 17 \\
\hline & 1.00 & 0.51 & 5 & 0.35 & 7 \\
\hline & 1.14 (EEMIR) & 0.66 & 4 & 0.50 & 5 \\
\hline \multirow[t]{4}{*}{$\mathrm{HF}$} & 0.50 (FEM) & 0.24 & 10 & 0.14 & 16 \\
\hline & 0.75 & 0.23 & 10 & 0.13 & 17 \\
\hline & 1.00 & 0.17 & 13 & 0.10 & 21 \\
\hline & 1.14 (EEMR) & 0.15 & 15 & 0.09 & 23 \\
\hline \multirow[t]{4}{*}{$\mathrm{LF}$} & 0.50 (FEM) & 0.18 & 13 & 0.10 & 21 \\
\hline & 0.75 & 0.16 & 14 & 0.09 & 23 \\
\hline & 1.00 & 0.12 & 17 & 0.07 & 29 \\
\hline & 1.14 (EEMR) & 0.11 & 20 & 0.06 & 33 \\
\hline
\end{tabular}

Tahin 1: Reproducing Kernel Particle Method $\gamma$-accuracy for the first-order wave equation in terms of phase and group speed. Results are presented for a range of refinement parameters and $N_{\gamma}$ has been rounded to the next highest integer.

\begin{tabular}{|c||c|c|c|c|}
\hline Method & $\mathrm{r}=0.50(\mathrm{FEM})$ & $\mathrm{r}=0.75$ & $\mathrm{r}=1.00$ & $\mathrm{r}=1.14($ EEMR) \\
\hline CF & $O\left(\Delta x^{4}\right)$ & $O\left(\Delta x^{2}\right)$ & $O\left(\Delta x^{8}\right)$ & $O\left(\Delta x^{2}\right)$ \\
\hline HF & $O\left(\Delta x^{2}\right)$ & $O\left(\Delta x^{2}\right)$ & $O\left(\Delta x^{2}\right)$ & $O\left(\Delta x^{2}\right)$ \\
\hline LF & $O\left(\Delta x^{2}\right)$ & $O\left(\Delta x^{2}\right)$ & $O\left(\Delta x^{2}\right)$ & $O\left(\Delta x^{2}\right)$ \\
\hline CT & N/A & $O\left(\Delta x^{2}\right)$ & $O\left(\Delta x^{4}\right)$ & $O\left(\Delta x^{2}\right)$ \\
\hline
\end{tabular}

Table 2: Reproducing Kernel Particle Method asymptotic convergence rates for the firstorder wave equation semi-discretizations and a range of refinement parameters. 


\begin{tabular}{|c|c|c|c|c|c|}
\hline \multirow[b]{2}{*}{ Method } & \multirow[b]{2}{*}{$r$} & \multicolumn{2}{|c|}{ Phase } & \multicolumn{2}{|c|}{ Group } \\
\hline & & $\overline{(2 \Delta x / \lambda)_{\gamma}}$ & $N_{\gamma}$ & $\overline{(2 \Delta x / \lambda)_{\gamma}}$ & $N_{\gamma}$ \\
\hline \multirow[t]{4}{*}{$\overline{\mathrm{CF}}$} & 0.50 (FEM) & 0.35 & 7 & 0.20 & 11 \\
\hline & 0.75 & $\overline{0.59}$ & 5 & 0.36 & 7 \\
\hline & 1.00 & 1.00 & 3 & 0.66 & 5 \\
\hline & 1.14 (EEMR) & 1.00 & 3 & 0.76 & 4 \\
\hline \multirow[t]{4}{*}{$\overline{\mathrm{CT}}$} & 0.50 (FEM) & $\mathrm{N} / \mathrm{A}$ & $\mathrm{N} / \mathrm{A}$ & $\overline{N / A}$ & N/A \\
\hline & 0.75 & 0.22 & 11 & 0.13 & 17 \\
\hline & 1.00 & 0.51 & 5 & 0.35 & 7 \\
\hline & 1.14 (EEMR) & 0.66 & 4 & 0.50 & 5 \\
\hline \multirow[t]{4}{*}{$\mathrm{HF}$} & 0.50 (FEM) & 0.68 & 4 & 0.46 & 6 \\
\hline & 0.75 & 0.36 & 7 & 0.21 & 11 \\
\hline & 1.00 & 0.24 & 10 & 0.14 & 16 \\
\hline & 1.14 (EEMR) & 0.21 & 11 & 0.12 & 18 \\
\hline \multirow[t]{4}{*}{ LF } & 0.50 (FEM) & 0.35 & 7 & 0.20 & 11 \\
\hline & $0.75 \cdots$ & 0.24 & 10 & 0.14 & 16 \\
\hline & 1.00 & 0.18 & 13 & 0.10 & 21 \\
\hline & 1.14 (EENR) & 0.15 & 14 & 0.09 & 24 \\
\hline
\end{tabular}

Table 3: Reproducing Kernel Particle Method $\gamma$-accuracy for the second-order wave equation in terms of phase and group speed. Results are presented for a range of refinement parameters and $N_{\gamma}$ has been rounded up to the next highest integer.

\begin{tabular}{|c||c|c|c|c|}
\hline Method & $\mathrm{r}=0.50$ (FEM) & $\mathrm{r}=0.75$ & $\mathrm{r}=1.00$ & $\mathrm{r}=1.14$ (EEMR) \\
\hline CF & $O\left(\Delta x^{2}\right)$ & $O\left(\Delta x^{2}\right)$ & $O\left(\Delta x^{6}\right)$ & $O\left(\Delta x^{2}\right)$ \\
\hline HF & $O\left(\Delta x^{4}\right)$ & $O\left(\Delta x^{2}\right)$ & $O\left(\Delta x^{2}\right)$ & $O\left(\Delta x^{2}\right)$ \\
\hline LF & $O\left(\Delta x^{2}\right)$ & $O\left(\Delta x^{2}\right)$ & $O\left(\Delta x^{2}\right)$ & $O\left(\Delta x^{2}\right)$ \\
\hline CT & N/A & $O\left(\Delta x^{2}\right)$ & $O\left(\Delta x^{4}\right)$ & $O\left(\Delta x^{2}\right)$ \\
\hline
\end{tabular}

Table 4: Reproducing Kernel Particle Method asymptotic convergence rates for the second-order wave equation semi-discretizations and a range of refinement parameters. 


\begin{tabular}{|c||c|c|c|}
\hline Method & $r$ & $(2 \Delta x / \lambda)_{\gamma}$ & $N_{\gamma}$ \\
\hline \hline \multirow{4}{*}{ CF } & 0.50 (FEM) & 0.24 & 10 \\
\cline { 2 - 4 } & 0.75 & 0.45 & 6 \\
\cline { 2 - 4 } & 1.00 & 0.81 & 4 \\
\cline { 2 - 4 } & 1.14 (EEMR) & 0.91 & 4 \\
\hline \hline \multirow{4}{*}{ CT } & 0.50 (FEM) & N/A & N/A \\
\cline { 2 - 4 } & 0.75 & 0.16 & 14 \\
\cline { 2 - 4 } & 1.00 & 0.44 & 6 \\
\cline { 2 - 4 } & 1.14 (EEMR) & 0.61 & 5 \\
\hline \hline \multirow{4}{*}{ HF } & 0.50 (FEM) & 0.58 & 5 \\
\cline { 2 - 4 } & 0.75 & 0.25 & 9 \\
\cline { 2 - 4 } & 1.00 & 0.17 & 13 \\
\cline { 2 - 4 } & 1.14 (EEMR) & 0.15 & 14 \\
\hline \hline \multirow{3}{*}{ LF } & 0.50 (FEMI) & 0.25 & 9 \\
\cline { 2 - 4 } & 0.75 & 0.17 & 13 \\
\cline { 2 - 4 } & 1.00 & 0.12 & 17 \\
\cline { 2 - 4 } & 1.14 (EEMR) & 0.11 & 20 \\
\hline
\end{tabular}

Table 5: Reproducing Kernel Particle Method $\gamma$-accuracy for the parabolic PDE in terms of numerical diffusivity. Results are presented for a range of refinement parameters and $N_{\gamma}$ has been rounded to the next highest integer.

\begin{tabular}{|c||c|c|c|c|}
\hline Method & $\mathrm{r}=0.50$ (FEM) & $\mathrm{r}=0.75$ & $\mathrm{r}=1.00$ & $\mathrm{r}=1.14$ (EEMR) \\
\hline CF & $O\left(\Delta x^{2}\right)$ & $O\left(\Delta x^{2}\right)$ & $O\left(\Delta x^{6}\right)$ & $O\left(\Delta x^{2}\right)$ \\
\hline HF & $O\left(\Delta x^{4}\right)$ & $O\left(\Delta x^{2}\right)$ & $O\left(\Delta x^{2}\right)$ & $O\left(\Delta x^{2}\right)$ \\
\hline LF & $O\left(\Delta x^{2}\right)$ & $O\left(\Delta x^{2}\right)$ & $O\left(\Delta x^{2}\right)$ & $O\left(\Delta x^{2}\right)$ \\
\hline CT & N/A & $O(\Delta x)^{2}$ & $O\left(\Delta x^{4}\right)$ & $O\left(\Delta x^{2}\right)$ \\
\hline
\end{tabular}

Table 6: Reproducing Kernel Particle Method asymptotic convergence rates for the parabolic PDE semi-discretizations and a range of refinement parameters. 